\title{
Microscopic polyangiitis in an unassuming child with arthritis: A case report
}

\author{
Neha Da Rocha ${ }^{1}$, Prashant Prabhu ${ }^{2}$, Gordon Politano Martins ${ }^{1}$, Annely Dlima ${ }^{2}$, Maria Silveira ${ }^{3}$ \\ From ${ }^{1}$ Junior Resident, ${ }^{2}$ Lecturer, ${ }^{3} \mathrm{HOD}$, Department of Pediatrics, Goa Medical College, Bambolim, Goa
}

\begin{abstract}
Microscopic polyangiitis is a rare vasculitis in childhood that primarily affects the kidneys and lungs. Here, we present the case of a 9-year-old boy who presented with fever and arthritis of the left knee and ankle. Investigations incidentally revealed high creatinine and microscopic hematuria. Perinuclear anti-neutrophil cytoplasmic antibody (pANCA) and renal biopsy were suggestive of microscopic polyangiitis. The patient was given steroids and cyclophosphamide; however, he expired of a catastrophic diffuse alveolar hemorrhage. This case highlights the need to keep ANCA-associated vasculitis in mind during workup for hematuria and unexplained acute kidney injury to aid in early diagnosis and management of the underlying condition.
\end{abstract}

Key words: Arthritis, Microscopic polyangiitis, pANCA

$\mathrm{M}$ icroscopic polyangiitis (MPA) is an idiopathic systemic vasculitis that is characterized by the inflammation of predominantly small-caliber blood vessels and the presence of antineutrophil cytoplasmic autoantibodies (ANCA). Because of its association with ANCA, it is often classified as ANCA-associated vasculitis, others being Wegener's granulomatosis (WG) and ChurgStrauss syndrome (CSS) [1]. Although it has multisystem involvement, MPA typically causes both pulmonary capillaritis and glomerulonephritis and hence, MPA is considered as an etiology of the pulmonary-renal syndrome, others being anti-glomerular basement membrane antibody disease (anti-GBM), systemic lupus erythematosus, and WG [1]. However, MPA is seen rarely in the pediatric age group, and data regarding its incidence in children is scarce $[2,3]$.

We are reporting the case of a child who presented with fever and arthritis, who was diagnosed to have MPA, but despite prompt immunosuppressive treatment, he succumbed to the pulmonary complications of the disease.

\section{CASE REPORT}

A 9-year-old boy was referred to our outpatient department with complaints of left ankle swelling, left knee pain, and intermittent fever $\left(100-101^{\circ} \mathrm{F}\right)$ over the past 4 months. He also complained of fatigue, malaise, and a significant drop in weight $(2.5 \mathrm{~kg})$ over the past four months. There was no history of cough, nasal discharge, breathlessness, blood in

\section{Access this article online}

Received - 30 April 2021

Initial Review - 15 May 2021

Accepted - 09 June 2021

DOI: $10.32677 / I J C R .2021 . v 07.106 .010$ urine, periorbital puffiness, mucocutaneous ulcers, or any significant drug intake.

The child was developmentally normal. He was admitted 2 months prior with gastrointestinal (GI) complaints of vomiting and abdominal pain. Isolated microscopic hematuria was noted during a urine routine examination performed for the GI symptoms. He was treated symptomatically and discharged after 4 days.

On general examination, Body Mass Index (BMI) was $<3^{\text {rd }}$ centile. The blood pressure was noted to be between 90 and $95^{\text {th }}$ centile with no tachycardia, or tachypnea. He had severe pallor, minimal bilateral pitting edema till shin, and left knee arthritis. Slit-lamp examination showed no evidence of uveitis. There was no organomegaly, no wheezing, and good air entry on auscultation. The rest of the systemic examination was normal. Urine output was $2-3 \mathrm{ml} / \mathrm{kg} /$ day.

Hematological investigations revealed anemia (hemoglobin$4.9 \mathrm{~g} / \mathrm{dl}$ ) with a total count of 10600 and a differential count of $\mathrm{N} 84 / \mathrm{L} 2 / \mathrm{E} 11$. Liver function tests showed bilirubin $0.4 / 0.2 \mathrm{mg} / \mathrm{dL}$, SGOT $-8 \mathrm{IU} / \mathrm{dl}$, SGPT - $105 \mathrm{IU} / \mathrm{dl}$ while renal function tests revealed blood Urea-74 $\mathrm{mg} / \mathrm{dL}$, and creatinine-2.6 $\mathrm{mg} / \mathrm{dL}$. Electrolytes were normal with fractional excretion of sodium (FeNa) - 2.5 signifying intrinsic renal injury. The urine routine showed numerous red blood cells (RBCs) and albumin of 4+ which when quantified revealed a urine protein creatinine ratio of 5.3 suggestive of nephrotic range proteinuria. Blood and urine cultures were sterile. To rule out acute glomerulonephritis (AGN) were normal. Rheumatoid Arthritis (RA) factor was negative. Antistreptolysin O (ASO) titers were normal. Erythrocyte sedimentation rate (ESR) was 66, C-reactive protein (CRP) was positive, and anti-nuclear Antibody (ANA) was negative. The

Correspondence to: Gordon Politano Martins, H.no 217A, Bironaik Ward, Orlim, Salcete, Goa, India. E-mail: gordonmartins_105@yahoo.com

(C) 2021 Creative Commons Attribution-NonCommercial 4.0 International License (CC BY-NC-ND 4.0). 
coagulation profile (prothrombin time, INR, and activated partial thromboplastin time) was normal.

Abdominal USG was performed which showed that both kidneys were mildly enlarged with raised parenchymal echogenicity and accentuated cortico-medullary differentiation with restricted movement on respiration. Minimal ascites were also present. Renal Doppler was normal. Chest X-ray showed no abnormalities. 2D ECHO showed a structurally normal heart with an ejection fraction of $60 \%$. The child received one packed RBC transfusion at $10 \mathrm{ml} / \mathrm{kg}$. Blood pressure and urine output were closely monitored in the hospital.

On day 7 of admission, the child developed one episode of generalized tonic-clinic seizures. Blood workup revealed a rise in urea to 92 and creatinine to 3.3. Blood pressure was found to be beyond the $99^{\text {th }}$ centile. Computed tomography (CT) of the brain showed a gliotic area in the right lentiform nucleus. In view of the clinical deterioration and worsening renal functions, the child was shifted to the Pediatric intensive care unit (PICU) and subjected to hemodialysis. Simultaneously, he was started on labetalol infusion for blood pressure control. Further blood workup revealed pANCA-positive (titer 1:100) and cANCA-negative.

A renal biopsy was performed. Histopathology and immunofluorescence of the kidney tissue (Figs. 1a-d) revealed necrotizing and crescentic glomerulonephritis in the sclerotic phase. There was acute tubular injury, interstitial fibrosis $(<25 \%)$, mild arteriosclerosis, and the absence of immunoglobin deposits. The primary diagnosis given was pauci-immune crescentic glomerulonephritis but based on the investigations, a diagnosis of Microscopic Polyangiitis (MPA) was made.

The child was given three pulse doses of intravenous methylprednisolone followed by daily oral prednisolone and one intravenous cyclophosphamide pulse dose. However, he continued to require hemodialysis every $2-3$ days. On the $26^{\text {th }}$ day of admission, the child developed respiratory distress and was started on humidified high-flow nasal cannula. Chest X-ray revealed fluffy perihilar shadows suggestive of pulmonary edema or diffuse pulmonary hemorrhage (Fig. 2). The child's distress progressively worsened requiring mechanical ventilation on the same day. Within a day, the child succumbed following a massive bout of expressed pulmonary haemorrhage.

\section{DISCUSSION}

Microscopic polyangiitis is a rare ANCA-associated vasculitis. It is defined by the Chapel Hill Consensus in 2012 as a "necrotizing vasculitis, with few or no immune deposits, predominantly affecting small vessels. Necrotizing glomerulonephritis is very common. Pulmonary capillaritis often occurs. Granulomatous inflammation is absent" [4]. Epidemiological data on microscopic polyangiitis in childhood are scarce. A study from Sweden shows the annual incidence is 0.7 per million [5] while another study from London claims that the incidence of MPA has not been established in the pediatric population [6].

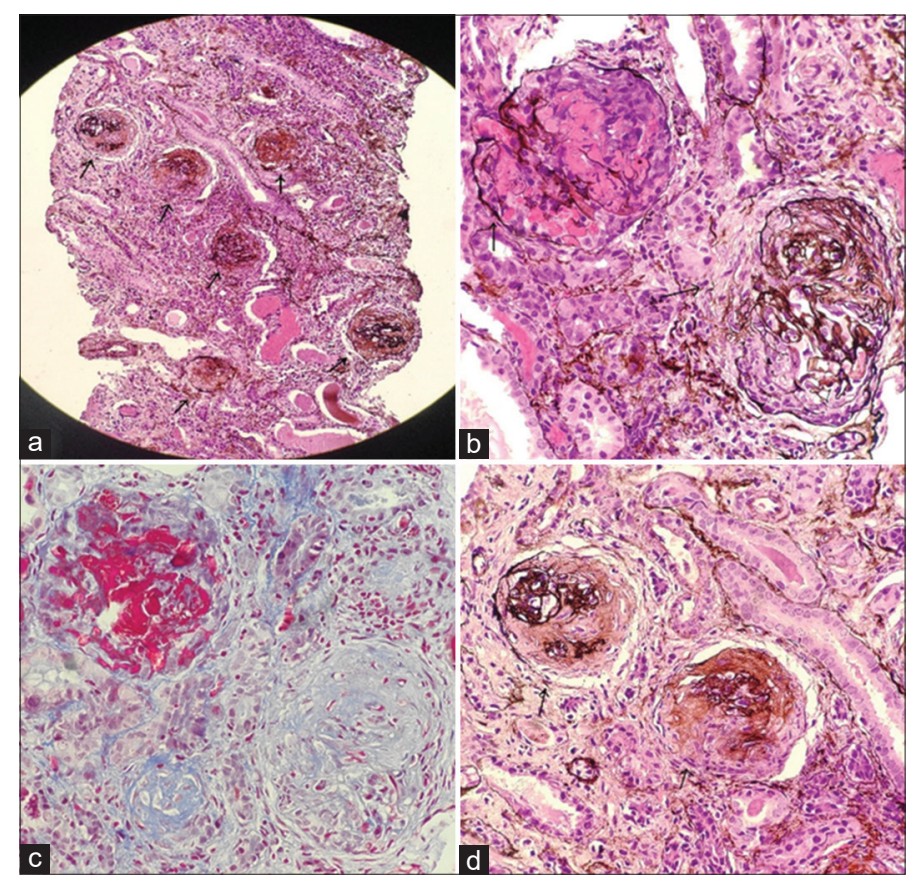

Figure 1: (a) Low magnification reveals 6 glomeruli possessing circumferential crescents (PASM stain); (b) High magnification reveals 2 glomeruli. Glomerulus on the left side shows circumferential active cellular crescent with tuft necrosis in the underlying tuft. Glomerulus on the right side shows fibrocellular crescent with rupture of capillary walls (PASM stain); (c) Same area shown in (b) stained with Mason Trichrome stain, reveals fibrinoid necrosis within the glomerular tufts on the left side; (d) High magnification reveals 2 glomeruli possessing circumferential fibrous crescents (PASM stain)

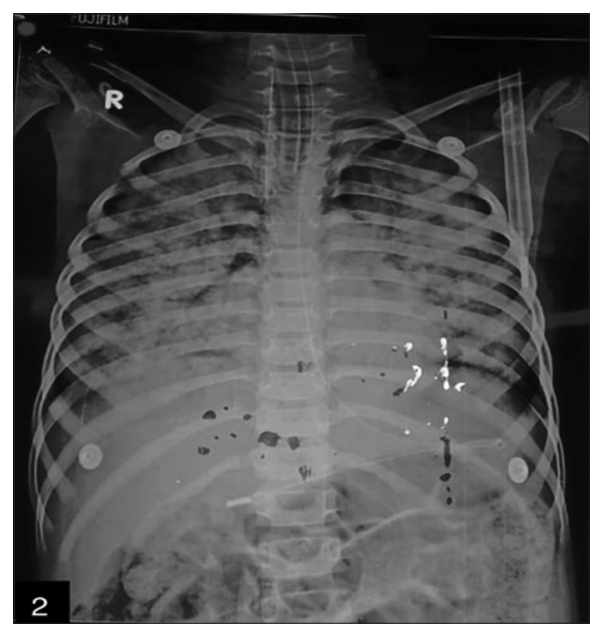

Figure 2: Chest X-ray (AP view) showing diffuse alveolar shadows, suggestive of pulmonary hemorrhage

Renal involvement is the most frequent manifestation on presentation, followed by systemic features, musculoskeletal, cutaneous, lower respiratory tract, and gastrointestinal involvement in the absence of granulomatous lesions [7,8]. In our case, the child presented with arthritis and vague systemic complaints and was incidentally found to have hematuria. The renal involvement that occurs in childhood ultimately progresses to chronic kidney disease in adulthood [9]. Diffuse alveolar hemorrhage can be present in about $40 \%$ of patients $[10,11]$. ANCAs against myeloperoxidase (MPO)(MPO-ANCA) remain the hallmark of the disease [9]. 
Renal biopsy is the gold standard for diagnosis and is an important predictor of renal outcome. ANCA-associated glomerulonephritis is characterized by pauci-immune immunofluorescence, necrotizing, and crescentic lesions in the affected glomeruli at light microscopy [2]. High-dose glucocorticoids and cyclophosphamide (either oral or intravenous) for 3-6 months are considered the gold standard of treatment also in children [2,12]. The prognosis often remains guarded [9].

\section{CONCLUSION}

Our case report highlights the importance of thoroughly evaluating unexplained hematuria and keeping ANCA-associated vasculitis in the differential diagnosis in a case of unexplained acute kidney injury. It also reminds us to be prepared for the catastrophic complications like alveolar hemorrhage that are associated with the disease.

\section{REFERENCES}

1. Chung SA, Seo P. Microscopic polyangiitis. Rheum Dis Clin North Am 2010;36:545-58.

2. Calatroni M, Oliva E, Gianfreda D, Gregorini G, Allinovi M, Ramirez GA, et al. ANCA-associated vasculitis in childhood: Recent advances. Ital J Pediatr 2017;43:1-9.

3. Arulkumaran N, Jawad S, Smith SW, Harper L, Brogan P, Pusey CD, et al. Long-term outcome of paediatric patients with ANCA vasculitis. Pediatr Rheumatol 2011;9:1-7.

4. Jennette JC, Falk RJ, Bacon PA, Basu N, Cid MC, Ferrario F, et al. 2012
Revised International Chapel Hill consensus conference nomenclature of vasculitides. Arthritis Rheum 2013;65:1-11.

5. Mossberg M, Segelmark M, Kahn R, Englund M, Mohammad AJ. Epidemiology of primary systemic vasculitis in children: A populationbased study from Southern Sweden. Scand J Rheumatol 2018;47:295-302.

6. Stegmayr BG, Gothefors L, Malmer B, Muller Wiefel DE, Nilsson K, Sundelin B. Wegener granulomatosis in children and young adults. A case study of ten patients. Pediatr Nephrol 2000;14:208-13.

7. Iudici M, Quartier P, Terrier B, Mouthon L, Guillevin L, Puéchal X. Childhood-onset granulomatosis with polyangiitis and microscopic polyangiitis: Systematic review and meta-analysis. Orphanet J Rare Dis 2016;11:141

8. Yu F, Huang JP, Zou WZ, Zhao MH. The clinical features of anti-neutrophil cytoplasmic antibody- associated systemic vasculitis in Chinese children. Pediatric Nephrol 2006;21:497-502.

9. Jariwala MP, Laxer RM. Primary vasculitis in childhood: GPA and MPA in childhood. Front Pediatr 2018;6:1-11.

10. Avner ED, Harmon WE, Niaudet P, Yoshikawa N, Emma F, Goldstein S. Pediatric Nephrology. $7^{\text {th }}$ ed. Heidelberg: Springer; 2016.

11. Cabral DA, Canter DL, Muscal E, Nanda K, Wahezi DM, Spalding SJ, et al. Comparing presenting clinical features in 48 children with microscopic polyangiitis to 183 children who have granulomatosis with polyangiitis (Wegener's): An Archive cohort study. Arthritis Rheumatol 2016;68:2514-26.

12. Eleftheriou D, Melo M, Marks SD, Tullus K, Sills J, Cleary G, et al. Biologic therapy in primary systemic vasculitis of the young. Rheumatology (Oxford) 2009;48:978-86.

Funding: None; Conflicts of Interest: None Stated.

How to cite this article: Da Rocha N, Prabhu P, Martins GP, Dlima A, Silveira M. Microscopic polyangiitis in an unassuming child with arthritis: A case report. Indian J Case Reports. 2021;7(6):251-253. 